\title{
Breast Segmentation with Pectoral Muscle Suppression on Digital Mammograms
}

\author{
David Raba, Arnau Oliver, Joan Martí, Marta Peracaula, and Joan Espunya \\ Robotics and Computer Vision Group, University of Girona, Av. Santalo s/n \\ Ed. p-IV, 17071 Girona, Spain \\ \{draba, aoliver, joanm, martapb, jespunya\}@eia.udg.es \\ http://vicorob.udg.es
}

\begin{abstract}
Previous works on breast tissue identification and abnormalities detection notice that the feature extraction process is affected if the region processed is not well focused. Thereby, it is important to split the mammogram into interesting regions to achieve optimal breast parenchyma measurements, breast registration or to put into focus a technique when we search for abnormalities. In this paper, we review most of the relevant work that has been presented from 80's to nowadays. Secondly, an automated technique for segmenting a digital mammogram into breast region and background, with pectoral muscle suppression is presented.
\end{abstract}

\section{Introduction}

Worldwide, more than 700,000 women die of breast cancer annually and it is estimated that eight to twelve percent of women will develop breast cancer in their lifetime.

Every effort directed to improve early detection is needed. Therefore, many computer vision techniques applied to analysis of digital mammograms have been proposed. Most of them require an initial processing step that splits the image into interesting areas, such as the breast region, background and patient markings. For example, it is well known that information derived from mammographic parenchyma patterns provides one of the most robust signs of risk of developing breast cancer. The largest breast region to be processed, the more accurate the classification of tissue will be. Moreover, the segmentation method should be robust enough to handle a wide range of mammographic images obtained from different image acquisition systems.

This work is part of a larger project called HRIMAC based on the analysis of mammographic images following two different approaches: 1)A Computed Aided Detection platform, which processes the mammograms as a second reader looking for abnormalities using BI-RADS [1] classification, and 2)A featured Computer Aided Diagnosis, which works as a Content Based Image Retrieval System (CBIR). We provide a case with mammogram and personal data and the system retrieves a set of similar cases from the database. This result tries to be a new information source to support radiologist diagnose. In both features, 
the automatic breast segmentation into background and breast region without artifacts (directly exposed area, the patient identification information and lead markers), is a key objective to provide useful data to the computerized analysis.

In this paper, we will present some of these techniques following this classification: Histogram, Gradient, Polynomial Modelling and Classifier approaches. In section 2.1, we propose an automated method to segment the digital mammogram into breast region and background with a new pectoral muscle suppression technique. Finally experimental and summary conclusions will be presented.

\section{Works on Breast Region Segmentation}

The breast gross-segmentation have been treated widely. Table 1 shows the tendencies and distribution of methods from the firsts works to recent approaches.

Table 1. Classification of breast gross-segmentation proposals.

\begin{tabular}{|c|c|c|c|}
\hline Methods & 1980's & 1990's & 2000's \\
\hline Histogram & Hoyer79 [2] & Lau91 [3] & Masek00 [4] \\
& & Yin91 [5] & \\
& & Bick95 [6] & \\
& & Byng96 [7] & \\
\hline Gradient & Semmlow80 [9] & Méndez96 [10] & Zhou01 [11] \\
& & Abdel-Mottaleb96 [12] & \\
& & Morton96 [13] & \\
& & Karssemeijer97 [14] & \\
\hline Polynomial Modelling & & Stomatakis94 [15] & \\
& & Chandrasekhar96 [16] & \\
\hline Active Contours & & Goodsitt98 [17] & \\
\hline Classifiers & & Ojala99 [18] & Ferrari00 [19] \\
& & & McLoughlin00 [20] \\
& & Wirth04 [21] \\
\hline & & & Saha01 [23] \\
& & & Rickard03 [24] \\
& & & Wirth04 [25] \\
& & & \\
\hline
\end{tabular}

- Histogram based techniques. Probably one of the first attempts to separate the breast region was presented by Hoyer et al [2] and it was done using simple histogram thresholding. The works of Lau et al [3], as well as Yin et al [5], and Byng et al [7] used a simple thresholding to segment the breast from the background. The work of Bick et al [6] presents a combination of local thresholding, region growing and morphological filtering. Hein et al [8] propose their own global histogram thresholding, while Masek et al [4] proposed a local thresholding method. 
- Gradient based techniques. Breast region extraction techniques based on gradient have long been in use, since the early work of Semmlow et al [9], who by means of spatial filters and a Sobel edge detector obtains the breast boundary. Similarly, Méndez et al [10] use a two-level histogram threshold to obtain the breast region and oriented upwards, the region is then divided into three parts to track the boundary using the gradient. An evaluation of the quality of the segmentation is provided using the "accurate" or "near accurate" labels. They compare successfully their results to the work presented by Yin et al [5]. The work presented by Karssemeijer et al [14] takes advantage of a multiresolution scheme, processing in low-res and extrapolating the result. Using a global thresholding technique they obtain a preliminary region, which is processed using a 3x3 Sobel operator, and the pectoral muscle position is estimated via Hough transform. Abdel-Mottaleb et al [12] provide an scheme based on different thresholds to find the breast edge. Using the gradient of two images and its union they obtain a possible breast contour. They found the boundary in $98 \%$ of the 500 images tested. The segmentation presented by Morton et al [13] was another gradient based method. After subtracting the background via an initial threshold, an edge was found by a line-by-line gradient analysis. Zhou et al [11] presented an improvement of this last approach.

- Polynomial Modelling based techniques. An early method proposed by Stomatakis et al [15] was not a strict polynomial modelling. By means of an image preprocessing technique to enhance the response of non-dark pixels, a noise reduction process and a histogram threshold, they obtain the breast region, but the boundary is smoothed using Cubic B-splines and samples at fixed pixel intervals are extracted. Then a smooth curve is generated through cubic polynomial calculations. One of the firsts, effective and real polynomial modelling was presented by Chandrasekhar and Attikiouzel [16]. An initial threshold is used to approximate the breast region. Their method provides around 94\% acceptable results from 300 images from MIAS [27] mammogram database. A quadratic/cubic polynomial fitting method was proposed by Goodsitt et al [17] which is fitted by translation and rotation the axes.

- Active Contours based techniques. One of the firsts applications of the active contours on breast segmentation was presented by Ojala et al [18]. McLoughlin et al [20]. They apply a global threshold to obtain an initial result. They statistically model the breast with the pixels inside the region and a snake algorithm is applied to obtain the final boundary. On the other hand, Ferrari et al [19] propose a method that firstly enhances the image with a logarithmic transformation, and then an iterative technique (as the Lloyd-Max least-squares) is applied to find and optimal threshold. Finally, they use a B-Spline to approximate the boundary. Recently, Wirth et al [21] propose an active contour to segment the breast. The method obtains two preliminary regions using a convolution matrix to enhance the edges and a dual threshold obtained by different techniques. They obtain the control point for the snake with the comparison of the two regions. They evaluate the method over the MIAS database. 
- Classifiers based techniques. Lou et al [22], used a clustering approach to obtain an initial region, estimates the real boundary extrapolating and linking those detected points. Saha et al [23] use a scale-based fuzzy connectedness algorithm. Rickard et al [24] presents Self-organizing map, a type of unsupervised artificial neural network model. The method applied by Wirth et al [25] was a fuzzy segmentation and evaluates the results in terms of completeness and correctness comparing the images from the MIAS database with a gold standard manually generated. Recently, Tromans et al [26], use a mixture model to obtain a mathematical representation of the image background and the compressed parameters, combined with a Fourier model, using an Expectation Maximization algorithm.

Summarizing, the traditional histogram based method has provided good and quick results. This quality sometimes turns on weakness in difficult cases where can be enhanced with local histogram or gradient approaches. The polynomial modelling and active contours provide very good results with accurate profiles.

\section{$2.1 \quad$ Our Method}

Figure 1 shows a visual scheme of the proposed method. To achieve the segmentation we propose a "two-phase" based method. It combines an adaptive Histogram approach to separate the breast from the background (Phase A), and a selective region growing algorithm to obtain pectoral muscle suppression (Phase B).

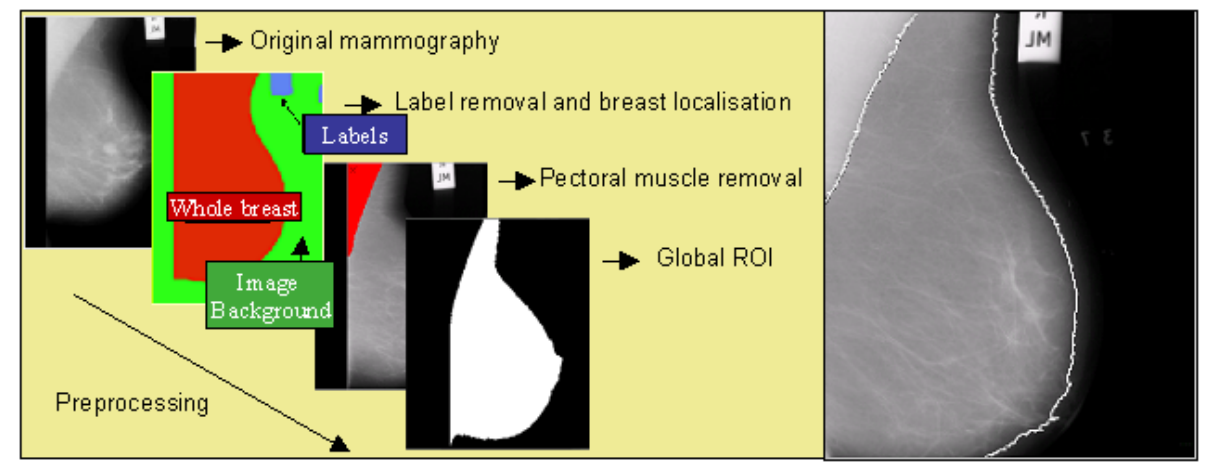

Fig. 1. Global Segmentation. Breast region extraction and pectoral muscle suppression.

Phase A. Breast Segmentation. Figure 1 shows the steps followed from the original mammogram to obtain the breast mask. A global histogram is calculated and smoothed with a gaussian operator. $\mathrm{N}$ consecutive percentage of bright pixels are tested to obtain $\mathrm{N}$ thresholds (ie. 10\%, 15\%, 20\% that in grey level means $220,210,200)$. Each value is used in order to threshold the image and obtain masks which are overlapped. The region defined by the boundary of the smallest 
threshold to the boundary of the largest one is statistically evaluated to calculate the mean of the grey level which is used as our final threshold value. The result of applying this threshold is a collection of different regions. The largest one is the union of the breast and the pectoral muscle. We extract this largest region using a Connected Component Labelling algorithm [28]. In Figure 1, the region of interest of the breast has been extracted from the pectoral muscle using the region growing algorithm described above. In the following section we introduce a new method to detect the pectoral muscle using a selective region growing approach.

Phase B. Extracting the Pectoral Muscle. This operation is important in mediolateral oblique view (MLO), where the pectoral muscle, slightly brighter compared to the rest of the breast tissue, can appear in the mammogram.

Previous work related to pectoral muscle suppression used Hough Transform [14, 29], assuming that the boundary between the pectoral muscle and the breast can be approximated by a straight line. Other related works are the one of Yam et al [30] whose work introduces a curvature component to the Hough estimation and the work presented by Ferrari et al [31] who propose a polynomial modelling of the pectoral muscle. The method we propose is inspired in the proposal of Georgsson [32] and in summary it follows the three steps:

1. Breast localisation and orientation. To classify the mammogram as right or left breast, we compare both sides of the breast profile, and using the curvature detected in each one, it is straightforward to determine the orientation.

2. Region growing intensity threshold estimation (RG). Once the orientation is known, a seed is placed inside the pectoral muscle (the first pixel of the non-curved side). A statistical region growing algorithm ( $\mathrm{RG}$ ) grows from this seed to fill the whole region of the pectoral muscle. A size restriction has been applied to avoid a wrong growing. When the limit of growing is exceeded, the growing criteria is corrected. This correction is estimated from the histogram of the previous region grown, progressively decreasing the initial value of the growing criteria. Then the $R G$ is restarted as shown in Figure 2. If a correct growing is not found in finite steps, the initial mask is provided as a result and the no existence of pectoral muscle is assumed.

3. Boundary refinement. Finally, the pectoral muscle is suppressed from the breast region, and a morphological operator is applied to refine the boundary.

\section{Experimental Results}

We have used the public database MiniMIAS [33] to test our method. It is a reduced version of the original MIAS Database (digitized at 50 micron pixel edge) that has been reduced to 200 micron pixel edge and clipped or padded so that every image is $1024 \times 1024$ pixels. 


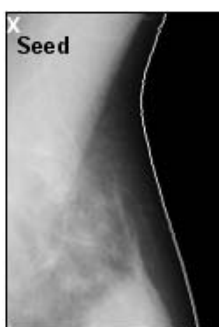

(a)

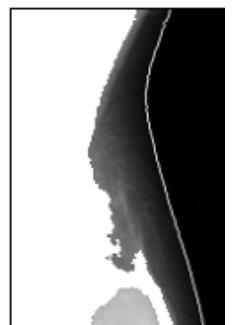

(b)

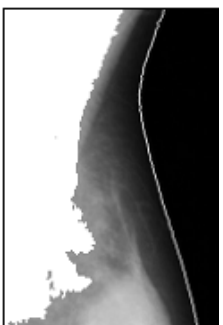

(c)

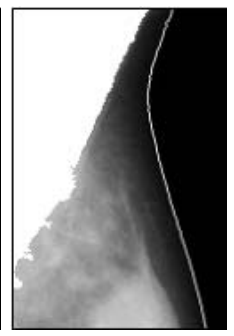

(d)

Fig. 2. Pectoral muscle removal. Region growing criteria correction. (a) original image,(b),(c) wrong RG (d) final correct RG.

Figure 3 shows three representative results. We have tested over 320 images, and we have obtained a $98 \%$ of "near accurate" results, which include the "accurate" results. About the muscle substraction, we have obtained a $86 \%$ of good extractions. Those results are obtained from a visual inspection of the images carried out by experienced radiologists and technicians trained with those kind of images. We should notice that some of them are a little bit over or under segmented. The behavior of the method shows an over-segmentation of the breast in cases with dense tissue, where the contrast between the muscle and the tissue is fuzzy. In that cases, our method rejects the muscle detection and provides the region obtained without suppressing the muscle as a final result. A possible solution could be to impose shape restrictions to the growing process. To summarize, the results obtained by the method show that it is a robust approach but it can be improved in terms of accuracy. Even so, we accept this method because it provides useful regions (there is no meaningful loss of information).

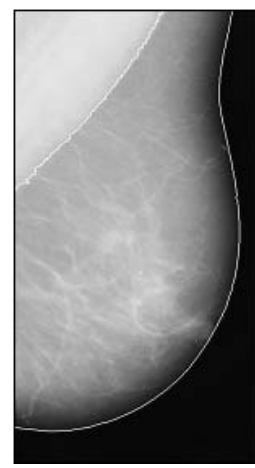

(a)

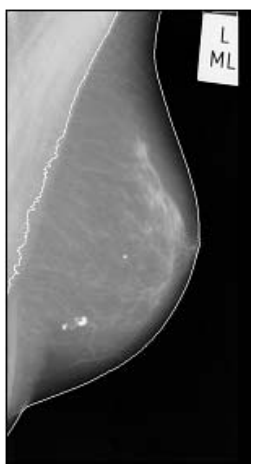

(b)

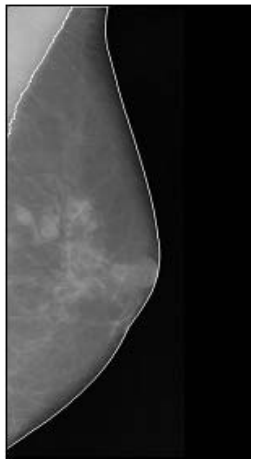

(c)

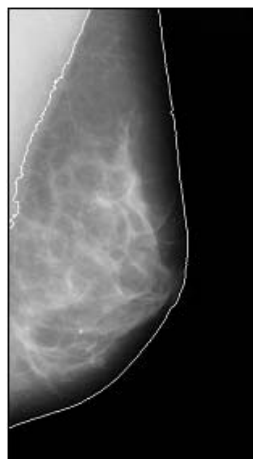

(d)

Fig. 3. An example of the performance of the presented approach on the segmentation of the profile of four different breasts. 


\section{Conclusions and Further Work}

The literature survey will be a useful resource for others researching in this area. A new method to segment the breast with pectoral muscle suppression has been presented. The results obtained over MiniMIAS database have shown a general good behavior. In this sense we will focus our efforts to enhance the method as we consider that is important to take some shape features into account to deal with the more accurate pectoral muscle suppression. The results have shown that problems with the image acquisition, background noise, artifacts and scratches could all influence the reliability of the algorithm.

\section{References}

1. Reston, V., ed.: Breast Imaging Reporting and Data System. 4th edn. American College of Radiology (1998)

2. Hoyer, A., Spiesberg, W.: Computerized mammogram processing. In: Phillips Technical Review. Volume 38. (1979) 347-355

3. Lau, T., Bischoff, W.: Automated detection of breast tumors using the asymmetry approach. In: Computers and Biomedical Research. Volume 24. (1991) 273-295

4. Masek, M., Attikiouzel, Y.: Skin-air interface extraction from mammograms using an automatic local thresholding algorithm. In: ICB, Brno, CR (2000) 204-206

5. Yin, F., Giger, M.: Computerized detection of masses in digital mammogram: analysis of bilateral subtraction images. In: Medical Physics. Volume 28. (1991) 955-963

6. Bick, U., Giger, M.: Automated segmentation of digitized mammograms. In: Academic Radiology. Volume 2. (1995) 1-9

7. Byng, J., Boyd, N.: Automated analysis of mammographic densities. In: Medical Physics. Volume 41. (1996) 909-923

8. Hein, J., Kallargi, M.: Multiresolution wavelet approach for separating the breast region from the background in high resolution digital mammography. In: Digital Mammography, Nijmegen, Kluwer Academic Publishers (1998) 295-298

9. Semmlow, J., Shadagopappan, A.: A fully automated system for screening xeromammograms. In: Computers and Biomedical Reseach. Volume 13. (1980) 350-362

10. Méndez, A., Tahoces, P.: Automatic detection of breast border and nipple in digital mammograms. In: Computer Methods and Programs in Biomedicine. Volume 49. (1996) 253-262

11. Zhou, C., Chan, H.: Computerized image analysis: Estimation of breast density on mammograms. In: Med. Phys. Volume 28. (2001) 1056-1069

12. Abdel-Mottaleb, M., Carman, C.: Locating the boundary between the breast skin edge and the background in digitized mammograms. In: Digital Mammography. (1996) 467-470

13. Morton, A., Chan, H., Goodsitt, M.: Automated model-guided breast segmentation algorithm. In: Med Phys. (1996) 1107-1108

14. Karssemeijer, N., te Brake, G.: Combining single view features and asymmetry for detection of mass lesions. In: IWDM. (1998) 95-102

15. Stomatakis, E., Cairns, A.: A novel approach to aligning mammograms. In: Digital Mammography. (1994) 255-364 
16. Chandrasekhar, R., Attikiouzel, Y.: Gross segmentation of mammograms using a polynomial model. In: International Conference of the IEEE in Medicine and Biology Society. Volume 3. (1996) 1056-1058

17. Goodsitt, M., Chan, H.: Classification of compressed breast shapes for the design of equalisation filters in x-ray mammography. In: Medical Physics. Volume 25. (1998) 937-947

18. Ojala, T., Liang, J.: Interactive segmentation of the breast region from digitized mammograms with united snakes. Technical Report 315, Turku Centre for Computer Science (1999)

19. Ferrari, R., Rangayyan, R.: Segmentation of mammograms: Identification of the skin boundary and the pectoral muscle. In: IWDM. Volume 23. (2000)

20. McLoughlin, K., Bones, P.: Locating the breast-air boundary for a digital mammogram image. In: Image and Vision Computing. (2000)

21. Wirth, M., Stapinski, A.: Segmentation of the breast region in mammograms using snakes. In: Canadian Conference on Computer and Robot Vision. (2004) 385-392

22. Gauch, J.: Image segmentation and analysis via multiscale watershed hierarchies. In: IEEE Transactions on Image Processing. Volume 8. (1999) 69-79

23. Saha, P., Udupa, J.: Breast tissue density quantification via digitized mammograms. In: IEEE Transactions on Medical Imaging. Volume 20. (2001) 792-803

24. Rickard, H., Tourassi, G., Elmaghraby, A.: Self-organizing maps for masking mammography images. In: IEEE EMBS. (2003) 302-305

25. Wirth, M., Lyon, J., Nikitenko, D.: A fuzzy approach to segmenting the breast region in mammograms. In: IEEE FI. Volume 1. (2004) 474-479

26. Tromans, C., Brady, J., Warren, R.: A high accuracy technique for breast air boundary segmentation and the resulting improvement from its use in breast density estimation. In: IWDM. (2004) $17-18$

27. Ibrahim, N., Fujita, H.: Automated detection of clustered microcalcifications on mammograms: Cad system application to mias database. In: Physics in Medicine and Biology. Volume 42. (1997) 2577-2589

28. Sanz, L., Petkovic, D.: Machine vision algorithms for automated inspection of thin-film disk heads. Volume 10. (1988) 830-848

29. Kwok, S., Chandrasekhar, R., Attikiouzel, Y.: Automatic pectoral muscle segmentation on mammograms by straight line estimation and cliff detection. In: IIS Conference. (2001) 67-72

30. Yam, M., Brady, M.: Three-dimensional reconstruction of microcalcifications clusters from two mammographic views. In: Proc Medical Image. Volume 20. (2001) 479-489

31. Ferrari, R., Rangayyan, R.: Automatic identification of the pectoral muscle in mammograms. In: IEEE Transactions on Medical Imaging. Volume 23. (2004) 232-245

32. Georgsson, F.: Algorithms and Techniques for Computer Aided Mammographic Screening. PhD thesis, UMINF-01.15, Umeå University, Sweden (2001)

33. Suckling, J., Parker, J.: The mammographic images analysis society digital mammogram database. In: Exerpta Medica. International Congress Series. Volume 1069. (1994) 375-378 